\title{
Exploration de l'imaginaire des professeurs des écoles : repères méthodologiques
}

\author{
Exploração do imaginário dos professores das escolas: \\ apontamentos metodológicos
}
Exploration of the imaginary of teachers in schools: methodological notes

\section{Marie-Cécile Charrier et Bertrand Bergier*}

\section{Résumé}

Cet article présente les fondements épistémologiques et méthodologiques d'une recherche en sciences de l'éducation, dont le but est l'exploration de l'imaginaire de professeurs des écoles dans un contexte d'apprenance. La démarche, qui se veut compréhensive, soulève des questions d'objectivié/subjectivité pour satisfaire aux exigences de scientificité dont son caractère qualitatif ne l'exempte pas. C'est dans une réflexion sur le langage, envisagé comme compromis entre structuralisme et herméneutique, que le chercheur trouvera ses outils d'investigation et d'analyse:

* MCC: Doutora em educação, e-mail:marie-cecile.charrier@orange.fr BB: Doutor em sociologia, e-mail: bertrand.bergier@uco.fr 
entretien semi-directif sur la formation initiale et continue, Archétype-Test à 9 éléments de Yves Durand (2005), réalisé à la suite des Structures Anthropologiques de l'Imaginaire de Durand, G. (1992). Nous verrons comment l'analyse du symbolisme des termes de l'entretien, combinée au micro-univers défini par l'AT.9, permet de définir chaque sujet comme un héros, personnage principal d'une histoire mythique, celle de l'apprenance esquissée dans une autre étape de la recherche.

Mots-clés: Imaginaire de professeurs. Epistémologie. Herméneutique. Structures anthropologiques de l'imaginaire.

\section{Resumo}

Este artigo apresenta os fundamentos epistemológicos e metodológicos de uma pesquisa em ciências da educação, cujo objetivo é a exploração do imaginário de professores das escolas em um contexto de aprendizagem. O desenvolvimento, que se pretende compreensivo, destaca as questões de objetividade/subjetividade para satisfazer as exigências de cientificidade que não excluem seu caráter qualitativo. No quadro de uma reflexão sobre a linguagem, considerada como compromisso entre o estruturalismo e a hermenêutica, $o$ pesquisador encontrará seus instrumentos de investigação e de análise : entrevista semi-diretiva sobre a formação inicial e continuada, Arquétipo-teste construído de 9 elementos de Yves Durand (2005), realizada após as Estruturas Antropológicas do Imaginário, de G. Durand (1992). Veremos como a análise do simbolismo dos termos de entrevista, combinada ao micro-universo definido pelo AT.9, permite definir cada sujeito como um heroi, personagem principal de uma história mítica, aquela da aprendizagem esboçada em uma outra etapa da pesquisa.

Palavras-chave: Imaginário de professores. Epistemologia. Hermenêutica. Estruturas antropológicas do imaginário.

\section{Abstract}

This paper presents the epistemological and methodological foundations of a research in education sciences, aimed at the exploration of the imaginary of the schools teachers in a learning context. The development, which intends to be comprehensive, highlights the 
issues of objectivity/subjectivity to meet the scientific requirements which does not exclude its qualitative character. On a reflection about language, considered as a compromise between the structuralism and hermeneutics, the researcher finds his research and analysis instrument: semi-directive interview on initial and continuing training, Archetype test constructed on 9 elements of Durand, Yves (2005), performed after the anthropological structures of imaginary of Durand, G. (1992). We will see how the analysis of the symbolism of the terms of appointment, combined to micro-universe defined by AT.9, allows us to define each person as a hero, main character of a mythical story, that one of learning outlined in another stage of the research.

Keywords: Imaginary of teachers. Epistemology. Hermeneutics. Anthropological structures of imaginary.

\section{Introduction}

Cet article prolonge une recherche ayant pour titre Imaginaire des professeurs des écoles et apprenance " (CHARRIER,harrier 2012). Celle-ci fait vivre ensemble au moins trois domaines qui, à la fois en composent le terreau et les bornes (la carte), et déterminent une topique majeure (la boussole) (FABRE, 2011), entrelacs et jeu des particularités relatives à chacun des champs sollicités. Il s'agit d'un rapport au monde - par l'imaginaire -, d'un rapport au savoir - de type apprenance -, dans un milieu professionnel, celui des professeurs des écoles.

Nous nous proposons dans cet écrit de nous attarder sur la méthodologie mise en œuvre, son enracinement épistémologique et, partant, de cerner la disposition de recherche du chercheur, son intelligibilité du réel. Pour cela, nous nous intéresserons de manière privilégiée à la place du sujet-chercheur, invité à jongler avec son intuition et sa quête de repères au service d'une démarche qu'il veut « compréhensive ».

Après avoir brièvement, mais fermement, envisagé l'imaginaire comme premier dans cette recherche, nous verrons - après Ricoeur 
- comment le langage, redéfini entre structuralisme et herméneutique, s'impose comme le lieu même de la " compréhension ».

Nous montrerons alors comment le philosophe G. Durand et le psychologue Y. Durand parviennent à élaborer une « carte-repère » du monde multivoque des images. Le premier classe les constellations d'images pour définir "les Structures Anthropologiques de l'Imaginaire »(DURAND, G., 1992). A la suite de ce travail, le second réalise un test révélateur des univers mythiques individuels. Il s'agit de l'Archétype-Test à 9 éléments (AT.9) (DURAND, Y., 1988, 2005).

Nous définirons ensuite l'apprenance (CARRE, 2005), (TROCMEFABRE, 1999). La notion que recouvre ce néologisme sera vue sous l'angle d'un comportement humain, susceptible d'évoquer, chez les individus, des images spécifiques, relatives à sa qualité de rapport au savoir de type autoformation (DUMAZEDIER, 2002 ; PINEAU, 1983).

Nous serons alors en mesure de décrire ce que nous avons appelé "les outils du langage pour comprendre ». Sont combinés d'entretiens semidirectifs, conduits auprès de 5 professeurs des écoles, sur leur rapport à l'apprentissage depuis la formation initiale jusqu'à ce jour, et la passation d'un AT.9, complétée par un retour du résultat auprès de chaque intéressé.

Enfin, les différents résultats obtenus nous permettront de tracer la silhouette de "héros contemporains ", évoluant dans un univers, l'apprenance, dont - dans un souci de cohérence épistémologique - on aura préalablement dégagé les composantes mythiques.

\section{Origine du questionnement}

Lorsque, "l'imaginaire » des sujets enquêtés est érigé en " objet » privilégié de recherche, le terrain devient on ne peut plus instable et mouvant, jonché qu'il est des enjeux et des jeux de subjectivité et d'objectivité, de structures et de rêveries éveillées (BACHELARD, 1942 ; 1943). Une critique, prompte à pointer les défauts de scientificité et de rigueur faites aux 
"sciences molles ", peine à reconnaître à cette "maîtresse d'erreur et de fausseté » (PASCAL, 1993) ses richesses d'expression.

Car, contrairement à "l'image " - qui envahit nos environnements privés et publics -, contrairement à "l'imagination »-qualité, voire compétence indispensable à l'investissement de nombre d'emplois -, «l'imaginaire » est un vocable qui ne fait florès ni dans la vie courante, ni dans la vie professionnelle. Il évoque plutôt le patrimoine culturel et nous le réservons à la nostalgie de l'enfance, aux souvenirs des histoires de princesses sauvées des griffes de terribles dragons par de preux chevaliers.

Nous nous situons sur autre plan et proposons, à la suite de G. Durand (1964), de définir l'imaginaire comme étant à la fois constitutif de l'Homme et élaborant une réponse à son angoisse existentielle de la linéarité du Temps fatal. Cet imaginaire constitue un réservoir d'images archétypales, lourdes de sens, qui nous agissent, le plus souvent à notre insu.

C'est là - sur ce terrain de l'imaginaire - qu'un travail en sciences humaines, qui cherche sa validité scientifique, peut achopper. Comment, en effet, prétendre à l'intelligibilité du réel, depuis les profondeurs de l'inconscient collectif? P. Ricoeur (1969, p.32) nous offre les prémices d'une réponse méthodologique lorsqu'il écrit : «le plan où la compréhension s'exerce est le langage ».

\section{Le langage, lieu de compréhension}

Le langage est à la fois "fermé » par sa structure, la linguistique (SAUSSURE, 1916), et infiniment " ouvert » par sa capacité à symboliser (RICOEUR, 1969). Une exploration de l'imaginaire pour la compréhension de comportements humains, pourrait déclarer forfait devant ce qui ressemble à une aporie. Nous verrons qu'il n'en est rien, qu'il s'agit plutôt d'une rencontre - celle de l'herméneutique et du structuralisme. 


\section{A propos de structuralisme}

Le structuralisme trouve son origine dans le « Cours de linguistique générale » de Ferdinand de Saussure (1916). L'adaptation de cette analyse structurale à différents domaines des sciences humaines la divise en deux courants, l'un dit «formel », l'autre "figuratif».

Le structuralisme formel est celui de l'anthropologue LeviStrauss (1949), du psychiatre et psychanalyste Lacan (1956), ainsi que des philosophes Foucault (1966) et Derrida (1967). Autant d'auteurs dont les travaux s'intéressent aux liens qui existent entre les éléments d'un système, sans prise en compte des sujets, ni du contexte, ni du sens, ni même de l'histoire. Les perturbations éventuelles ne mettent pas en péril la solidarité des éléments. L'exercice maximal de cette approche est la mathématisation. Ainsi en est-il des structures que Levi Strauss dégage des mythologies des amérindiens. De la même façon, les relations entre les signes du langage sont gérées par les règles de la grammaire et de la syntaxe pour former le système linguistique que F. de Saussure nous a livré.

De ce point de vue, le langage est un système de signes fermé à l'imaginaire.

C'est sans compter la duplicité et l'équivocité qui s'installent lorsque les lettres s'organisent en mots, en expressions, en phrases et en textes. Nombre de termes revêtent ainsi plusieurs sens, relevant chacun d'une topique spécifique. Avec la phrase, puis le texte, «le langage exprime quelque chose "(RICOEUR, 1969, p. 118). Un sujet peut agir dans un contexte, une " histoire " se dessiner. Une évolution devient possible. Le système s'ouvre, le langage devient figuratif, symbolique. Dans la mesure où le contexte permet plusieurs isotopies à la fois, "le langage dit autre chose en disant une chose " (RICOEUR, 1969, p. 140). Ainsi, on voit que le langage est aussi un système de signes ouverts à l'interprétation.

Mise sous "le régime de cette ouverture de l'univers des signes" (SANDERS, cité dans RICOEUR, 1969, p. 103), l'herméneutique invite au dévoilement du sens. 


\section{A propos d'herméneutique}

Selon la fonction que l'exégète donne à son interprétation d'un texte, l'herméneutique est dite réductive ou instaurative.

Les herméneutiques réductives donnent une valence négative à l'imaginaire. Elles tentent de réduire le symbole au signe, au symptôme. Chez Freud (1900), l'imaginaire est toujours suspect, symptomatique d'un dérèglement caché, de refoulement. Jung $(1964,1995)$ propose une valence plus positive en faisant de la représentation un moment de réussite dans la thérapie.

Mais c'est, entre autres, avec G. Durand (1964) que l'herméneutique se fait instaurative. Pour lui, le symbolisme de Jung, qui considère le symbole comme multivoque, est trop large. C'est avec le langage poétique de Bachelard et «sa rêverie » des quatre éléments (feu, air, terre et eau), que la phénoménologie s'imposera à lui comme moyen d'exploration de l'imaginaire, redonnant à celui-ci, ainsi qu'au mythe, toute sa valence positive. Avec le langage poétique, l'homme peut se situer

« au-delà de l'asséchante objectivité ou de l'engluante subjectivité » (DURAND, 1964, p. 73). En héritier de Bachelard et de sa notion de rêverie éveillée, G. Durand considère qu'il n'y a pas de coupure entre le rationnel et l'imaginaire. Le rationalisme n'est qu'une structure polarisante particulière du champ des images, parmi d'autres. Le sens propre qui conduit au concept, n'est qu'un symbole restreint, un cas particulier du sens figuré. L'imagination est une fonction symbolique facteur d'équilibre social, soit l'interrelation entre le psychisme individuel et le milieu culturel et social. D'où la notion de " trajet anthropologique de l'imaginaire » (DURAND, G., 1969), qui est échange incessant entre les pulsions subjectives et assimilatrices et le milieu qui impose ses contraintes. 


\section{L’herméneutique pour comprendre}

Pour Dilthey (dans RICOEUR, P., 1969, p. 201), il ne s'agit pas d'expliquer causalement un phénomène, mais de comprendre sa signification. En choisissant d'interpréter les « discours » recueillis auprès de sujets dont il observe un comportement particulier, le chercheur affirme une posture épistémologique qui réduit la distance qui les sépare de lui. Il n'en sort pas indemne, car écrit Dilthey - pour qui la vie était le concept majeur (dans RICOEUR, P., 1969, p.28) -, " Comprendre [par la voie de l'interprétation] n'est plus alors un mode de connaissance, mais un mode d'être, le mode de cet être qui existe en comprenant ».

Comprendre par l'interprétation nous fait pénétrer le monde foisonnant des archétypes, des symboles, des mythes et, partant, rencontrer le doute. Sans repères, l'herméneute risque de se perdre dans la multivocité quasi infinie des signes. Comme il sauverait notre homme de la noyade, Michel Fabre lui tend une "perche " aussi pertinente que séduisante, relevant elle-même de l'imagerie. Devant la difficulté d'éduquer dans un monde qu'il qualifie d' « héraclitéen » car sans cesse en mouvement, M. Fabre (2011, p. 7) file la métaphore spatiale de la carte et de la boussole. Le bagage des expériences vécues délimite et cartographie le territoire dans lequel l'apprenant évolue. Cependant, "aucune orientation [n'apparaît], à première vue du moins, plus légitime qu'une autre " (FABRE, 2011, p. 8). C'est pourquoi le sujet est invité à choisir une direction relativement à ses projets, lesquels lui servent ainsi de boussole. L'herméneute fait de même, qui choisit dans le cartulaire des images élaboré par G. Durand (DURAND, 1969) l'interprétation possible d'un symbole au regard d'une topique déterminée, celle de sa recherche.

\section{A la recherche de la « carte " du territoire de l’imaginaire}

Gilbert Durand a consacré une grande partie de son travail de philosophe à " cartographier » le champ de l'imaginaire. Il s'est inspiré 
d'auteurs, sus-nommés, dont les thèses reconnaissent aux images leur valence positive - en cela plutôt Jung, que Freud pour la psychanalyse -, des auteurs qui les considèrent non comme négation de la réalité mais comme son élargissement - avec le scientifique Bachelard et sa poétique du feu, de l'eau, de la terre et de l'air. Durand, G. (1992) assemble les images en essaims, en constellations, et réalise ainsi une classification selon trois schèmes d'action, qui correspondent aux constatations psycho-physiologiques de l'école de Léningrad (notamment celle de Betcherev) relatives aux réflexes dominants. Ainsi, la dominante posturale, ou tendance à se redresser, recouvre les notions de verticalité et d'horizontalité et compose le Régime Diurne de l'imaginaire; la dominante digestive, ou tendance à engloutir, correspond à la notion d'intimité et de descente, et appartient au Régime Nocturne de l'imaginaire, de même que la dominante copulative ou tendance à rythmer et copuler; Ces trois schèmes d'action correspondent également, aux trois groupes de structures repérées dans la classification psychologique des symboles (schizomorphes, synthétiques, mystiques).

Le schème se fonde sur un mouvement concret, il est la structure abstraite du mouvement. Il fait la jonction entre les gestes inconscients de la sensori-motricité, entre les dominantes réflexes et les représentations. Selon l'expression de G. Durand, ils « forment le squelette dynamique, le canevas fonctionnel de l'imagination "(DURAND, G., 1969, p. 61). Chaque schème représente donc la matrice d'une série d'archétypes et d'images variées: symboles ou concepts, images symboliques plus ou moins universelles ou plus ou moins codifiées par l'environnement socio-culturel. G. Durand considère les archétypes comme une substantification des schèmes au contact de l'environnement naturel et social (par exemple, au schème ascensionnel correspondent les archétypes du ciel, du sommet, du chef). Ils ont un caractère primordial, stable et universel, alors que les symboles sont plus malléables et polysémiques.

Le tout constitue un tableau isotopique qui rend compte des Structures Anthropologiques de l'Imaginaire dégagées par l'auteur.

Les Structures Anthropologiques de l'Imaginaire ont permis à G. Durand de réaliser deux formes d'herméneutiques instauratives : d'une 
part, la mythanalyse qui cherche les constantes figuratives de moments culturels dans une société, et, d'autre part, la mythocritique qui consiste à repérer les mythes inspirateurs d'œuvres picturales et littéraires (ex. "Le décor mythique de la Chartreuse de Parme », DURAND, G., 1961).

\section{Les univers mythiques individuels}

Avant de chercher à déterminer les univers mythiques individuels, il convient de définir le concept de "mythe » et sa fonction dans le monde de l'imaginaire tel que nous venons de le présenter.

Les mythes (DURAND, G., 1996) sont des « histoires » - ni contes, ni légendes - remplies de symboles, dont les personnages sont des Etres extra-ordinaires. Ils réalisent une forme de synthèse des différents termes de l'Imaginaire - archétypes, schèmes, symboles - en les organisant de manière dynamique dans des scénarii qui promeuvent doctrines religieuses comme récits légendaires des sociétés. Il s'agit de drames qui font tenir ensemble les espoirs et les terreurs des hommes. Les mythes traditionnels ont offert, et permis de transmettre, une compréhension de l'Humanité, de ses origines, de ses comportements et de ses grandes questions existentielles. Ils évoluent cycliquement, en six phases qui constituent ce que G. Durand appelle le "bassin sémantique ». De la modernité à la post-modernité, la société illustre cette évolution qui va de Prométhée - figure mythique du progrès - à Dionysos - figure emblématique de l'ouverture qui entraîne la perte des repères. D'une manière générale, le mythe est repérable en partie par le Nom.

Le psychologue Durand, Y., (1988 - 2005), disciple et collaborateur de G. Durand, a enrichi le travail de celui-ci en élaborant un test qui permet aux sujets d'exprimer leur imaginaire en univers mythiques sur la base de 9 archétypes soigneusement choisis, qui jouent le rôle " d'attracteurs mythiques": "une chute, une épée, un refuge, un monstre dévorant, quelque chose de cyclique (qui tourne, qui se reproduit ou qui progresse), un personnage, de l'eau, un animal (oiseau, poisson, reptile ou mammifère), du feu ». Il s'agit 
de l’"Archétype-Test à 9 éléments » ou AT.9. Le " langage » sollicité y est pluriel: un dessin est proposé qui relie les 9 symboles en une histoire; celleci fait l'objet d'un récit écrit (langage en tant que système linguistique), et d'un questionnaire explicatif, écrit, portant sur ce récit. Il est également demandé, pour chacun des archétypes, de préciser par écrit sa morphologie (ou représentation), son rôle et ce qu'il symbolise. L'AT.9 sert l'objectif de compréhension du chercheur en tant qu'il ne conduit pas à la définition de typologies d'individus et qu'il n'est ni causaliste, ni objectiviste, ni même définitif.

La quête de repères dans le monde de l'imaginaire nous a conduits à dévoiler ce que nous avons appelé " les supports langagiers pour comprendre ", soit les outils d'investigation de la recherche. Avant de décrire leur mise en œuvre, il nous faut définir la notion d'apprenance et en esquisser le mythe.

\section{Un comportement humain pour exemple : l’apprenance}

\section{La notion d'apprenance}

L'ordre des termes du titre de la recherche "Imaginaire des professeurs des écoles et apprenance " (CHARRIERharrier, 2012) reflète la construction de la thématique en question, sa problématique, ainsi que ses choix méthodologiques. Il s'agit bien en effet de considérer la question de l'imaginaire comme première par rapport à la notion d'apprenance.

Elan vital pour H. Trocmé-Fabre (1999), l'apprenance est pour Philippe Carré (2005, p. 108) « un ensemble durable de dispositions favorables à l'action d'apprendre dans toutes les situations formelles ou informelles, de façon expériencielle ou didactique, autodirigée ou non, intentionnelle ou fortuite ». Ces dispositions sont d'ordre cognitif, affectif et conatif. Le plan cognitif concerne les représentations de l'individu quant aux contenus d'apprentissage, ces représentations ayant - selon 
Giordan (1998) - une influence décisive sur l'apprentissage lui-même. D'un point de vue affectif, la disposition à apprendre s'appuie sur les émotions - positives - de l'apprenant face aux différents paramètres de la situation d'apprentissage que sont les contenus, les formateurs, les partenaires. Les dispositions à apprendre de type conatif sont d'ordre motivationnel. Elles se révèlent dans les choix et l'engagement du sujet. Dans les écrits de P. Carré (2005), nous pouvons repérer des indicateurs de ces différents champs de la personne - vouloir agir, savoir apprendre et pouvoir agir - qui, mis en lien, dessinent le profil d'apprenance du professionnel.

Sous son néologisme, l'apprenance reconnaît la capacité de l'adulte à apprendre, distingue celle-ci de celle des enfants, et témoigne des manières dont elle a été prise en compte depuis le début du XXème siècle notamment. Elle constitue la dernière version de la formation des adultes, dans une société dite " cognitive » (CEE, 1995) après avoir été "éducative » (DUMAZEDIER, 1978) et " pédagogique » (BEILLEROT, 1982), soumise aux économies de savoirs après avoir servi les économies industrielles.

Le «travailleur du savoir » (CARRE, 2005, p.24) qu'est le salarié de l'apprenance se voit confier deux missions, dans un objectif de croissance durable : son développement et celui de l'entreprise. Il peut les remplir grâce et à la condition de la formation tout au long et tout au large de la vie, que les institutions accompagnent par des mesures diverses comme le DIF, et que les technologies de l'information et de la communication favorisent. Le salarié est ainsi passé du droit à la formation à l'obligation de compétence.

La figure de l'Auteur (COHEN, 2005) se dessine, dans un monde qui s'ouvre. Cependant, la posture qui sous-entend une croissance durable, n'est probablement pas sans risque. Qu'en est-il de la motivation des adultes à se former, qui plus est de manière réitérée ? Les «bonnes intentions éducatives » (DUMAZEDIER, 1978) de la société de l'apprenance sont aux prises avec l'objectif de profit des entreprises. 


\section{Esquisse du mythe de l'apprenance}

C'est autour de la question du mythe que l'univers de l'imaginaire et celui de l'apprenance s'articulent. Ce rapport spécifique au savoir cristallise en effet certains espoirs comme certaines terreurs des hommes de notre siècle.

L'acte d'apprendre confronte les individus à l'angoisse de l'incertitude, angoisse de la perte des connaissances antérieures dans leur intégrité. Dans le langage courant, la « perte de connaissance » est la " petite mort » de l'évanouissement. Les savoirs nouveaux participent d'une re-naissance de l'individu. Avec les questions d'origine et de perte (SIBONY, 1991; KAËS, 1997) qui, on le voit, traversent la notion-même d'apprentissage, l'apprenance ne rappelle-t-elle pas le "drame agrolunaire " (DURAND, 1969) d'une part, et ces mythes des origines que les civilisations anciennes ont élaborés d'autre part? La réitération de l'exercice de déstabilisation/rééquilibration que l'apprenance impose évoque en outre la nécessité pour ces civilisations de revivre la scène de la création - quand il ne s'agit pas seulement de la rejouer dans un rituel.

L'apprenance est un drame qui met en scène et fait interagir l'imaginaire de la notion même avec ceux de la société et du sujet social. La société apprenante cherche à maîtriser le temps par la répétition cyclique de l'acte d'apprendre, rendu accessible à tous. La nouveauté et l'éphémère découpent indéfiniment le temps dans un objectif de progrès, selon un mouvement ascendant de nature héroïque. Les traits du héros-conquérant du savoir sont ceux d'un Auteur. Son imaginaire est plutôt synthétique, qui harmonise des capacités intellectuelles (donc plutôt héroïques) et affectives (donc plutôt mystiques).

Avec Durand, (2005), on peut cerner les contours d'un drame dans lequel la société fait figure d'actant-sujet, soumis à un actant-objet négatif dont les exigences de compétitivité et de croissance durable endossent le rôle. En la figure de l'Auteur, apprenant permanent, heureux, responsable et autonome, la société trouve un Allié - actant-objet positif - pour un avenir meilleur. Le temps devient maîtrisable, grâce à un mythe synthétique qui 
rappelle la forme "Drame de la Chute et de la Rédemption par un médiateur » (DURAND, 1969).

\section{Les supports « langagiers » pour comprendre}

Quel type d'Allié - apprenant tout au long et tout au large de la vie - chaque professeur des écoles campe-t-il pour aider son institution de tutelle, l'Education Nationale, à répondre à l'exigence de développement, de compétitivité et de croissance durable, à laquelle le monde du XXIème siècle la soumet.

\section{a) Choisir des outils d'investigation}

\section{Deux outils d'investigation}

Choisir des outils d'investigation pour élaborer une réponse à la question de recherche, c'est prendre en compte la nature du terrain qui la fait émerger, le champ théorique dans laquelle elle s'inscrit .... sans oublier les personnalités du sujet et du chercheur. "tout regard est croisement de celui du locuteur et de celui du ou des destinataires »(DURAND, G., 1996)? Mise au service d'une approche compréhensive, non causaliste, la méthodologie dont il est question ici cherche à révéler les réponses "imaginaires » de professeurs des écoles soumis à l'injonction d'apprenance de la société cognitive. Les outils méthodologiques sont choisis pour servir l'exploration de leur imaginaire individuel et leur rapport à ce type de savoir. La démarche - avant tout qualitative - est à visée compréhensive. Sans souci de représentativité d'une population, elle exclut le recours au questionnaire. Plus intéressé par des questions de «quoi » que de " pourquoi », le chercheur souhaite favoriser la production de discours personnels sur des parcours professionnels, récolter des impressions, des ressentis, des souhaits, liés à l'expérience présente ou imaginée. On peut postuler, après J.-L. Rinaudo (2004), que les discours recueillis, bien que ne répondant pas directement à 
des questions de formation tout au long de la vie - soient riches d'éléments de compréhension du rapport au savoir, lesquels seront révélés grâce aux outils d'analyse. S'agissant de la production de discours qualitatifs sur des parcours, le choix des outils d'investigation se porte, ici, sur la conduite d'entretiens semi-directifs. Cette approche s'intéressera aux symboles que livre le discours du Sujet sur la formation et la profession; quand il s'agit, conformément à une démarche herméneutique dite "eschatologique ", de "dévoiler l'essence de l'esprit à travers les avatars [...] de notre situation là, maintenant dans le monde " (RICOEUR, P., cité par DURAND, 1964). Si l'entretien semi-directif permet l'émergence d'une thématique de longue durée, les systèmes mythiques individuels des sujets seront plus facilement révélés par une seconde approche, plus ouvertement " psychologique ", celle de l'Archétype-Test à 9 éléments élaboré par Y. Durand (DURAND, Y., 1988, 2005), à partir des Structures Anthropologiques de l'imaginaire de G. Durand. Par ces deux voies, le chercheur repère d'éventuelles redondances symboliques car, si l'AT.9 - outil normé - ne propose pas d'alternative en termes d'analyse, il en va différemment de l'entretien semi-directif.

\section{Complémentarité des outils d'investigation}

S'agissant des deux chemins d'accès à l'imaginaire individuel que constituent l'analyse du symbolisme des termes et l'AT.9, plusieurs thèmes se dégagent, qui soulignent leur complémentarité: le langage, la temporalité, la dimension existentielle du sujet et la question du cadre.

\section{La complémentarité langagière}

Deux modes de production langagière sont sollicités : la voie orale par les entretiens et le retour de l'analyse AT.9 aux intéressés ; la voie écrite de type linguistique et narratif pour l'AT9, la voie écrite de type pictural pour la représentation des archétypes de l'AT9. 


\section{La temporalité}

Une ouvre AT9 est créée à l'occasion d'une rencontre entre 2 personnes »(DURAND, Y., 2005). La notion de rencontre fait écho à celle du trajet anthropologique, source de l'imaginaire individuel. Le rendez-vous de deux subjectivités, dans un contexte spatial et temporel particulier, donne de l'imaginaire du sujet un "instantané », qui ne se retrouvera jamais plus à l'identique. Si la question de la subjectivité des protagonistes vaut aussi pour les entretiens semi-directifs - conduits de façon à mettre en lumière le rapport des professeurs des écoles à la formation -, les discours produits, en revanche, déroulent le plus souvent une temporalité "longue ", qui traverse l'ensemble du parcours professionnel, voire du parcours scolaire. Les informations que livre le discours sont susceptibles de dessiner des tendances imaginaires stables sur l'ensemble d'un parcours, ou fluctuantes au gré des épisodes professionnels - comme une illustration du trajet anthropologique entre psychisme et intimations du milieu. Sauf à être réitéré, l'AT.9 ne peut produire ce type d'information.

\section{La dimension existentielle du sujet.}

Le but du chercheur "ce n'est pas l'idéologie d'un homme ou d'une femme qu'il s'agit de déterminer dans une perspective clinique, mais l'authenticité existentielle d'une personne avec sa souffrance et ses possibilités créatrices » (DURAND, Y., 2005). Les dimensions affectives et cognitives ainsi explorées par l"AT.9 constituent autant d'éléments que l'injonction à l'apprenance sollicite également et qui émergeront dans les entretiens. En cohérence avec la thématique de la recherche, les deux outils se donnent une chance de la servir. 


\section{La question du cadre}

En utilisant conjointement l'AT.9 de Y. Durand et le tableau isotopique des images de G. Durand, le chercheur tente de se donner un cadre tout en se libérant d'un autre.

L'analyse du symbolisme des termes d'un entretien, au regard des travaux de G. Durand, est fortement soumise à la subjectivité du chercheur. Elle peut, de fait, produire des interprétations plus ou moins " magiques ", plus ou moins éloignées de celles du sujet lui-même. En révélant leurs difficultés, ou propensions, à intégrer les différents archétypes embrayeurs de structures, les AT.9 révèlent aussi les tendances imaginaires des sujets. Un " principe d'antagonisme " (LUPASCO, S., 1967) régit le caractère systémique et dynamique de l'imaginaire et permet à chaque individu, dans des processus d'hétérogénéisation et d'homogénéisation des images, de résoudre l'angoisse de mort liée au temps qui passe.

Avec ce test, le psychologue Y. Durand propose ainsi une approche bi-polaire (héroïco-mystique) plutôt que monopolaire, comme le tableau isotopique des images de G. Durand nous invite à le faire. L'approche structurale du sociologue est nuancée par l'utilisation de l'Archétype-test à 9 éléments. Quand les processus d'actualisation/potentialisation dessinent des micro-univers qui rapprochent les tendances "pures » héroïque et mystique.

Analyser le symbolisme des termes du discours sur le parcours professionnel, c'est s'intéresser à la vie exprimée du sujet, à un récit de type existentiel, quand l'AT9 est une création imaginaire très normée. La personnalité et la sensibilité du sujet profitent de la durée d'un entretien et de son caractère " semi-libre " pour, progressivement, se déployer et offrir des « redondances » qui constituent autant « d'indices » de son imaginaire. "Sortir du cadre » s'est concrètement révélé fructueux dans la conduite même des entretiens : lorsque le micro est coupé, l'interlocuteur livre parfois ce qui vient du plus profond de lui-même et que la durée du retour sur soi a rapproché de la surface dicible. Quand les acquis nocturnes de l'expérience parviennent à voir le jour (PINEAU, 1983) 
Les approches de G. Durand et de Y. Durand se nuancent et s'enrichissent. Si l'analyse selon $G$. Durand fait courir au chercheur le risque de l'interprétation abusive, elle lui permet aussi de " lire " l'imaginaire des sujets dans une temporalité longue. De la même façon, si l'analyse par l'AT9 de Y. Durand, - dans la mesure où celui-ci n'est pas réitéré comme c'est le cas ici -, fige un imaginaire dans une temporalité très courte, elle permet également d'en cadrer l'interprétation.

\section{b) Mise en ouvre des outils d'investigation et analyse}

Le terrain

La démarche, essentiellement qualitative, n'implique pas la rencontre d'une véritable population. Dans ce cas, "le critère de sélection de ces personnes est généralement la diversité maximale des profils en regard du problème étudié. »(QUIVY, R., VAN CAMPENHOUDT, L., 1995). La redondance des informations, et la difficulté à trouver de nouveaux visages de professionnels, signeront la limite de l'investigation. La constitution de l'échantillon croise des critères de genre, de prise de responsabilité et d'ancienneté dans la profession, de parcours professionnel et de situation familiale. Ainsi, sont approchés,

- Des professionnels exerçant actuellement - ou ayant exercé - les fonctions de chef d'établissement et/ou de Maître Associé à la Formation; et des professionnels n'exerçant pas, et n'ayant jamais exercé, ce type de responsabilité dans l'institution;

- Des professionnels justifiant d'une ancienneté minimum de 15 ans, afin d'offrir un regard suffisamment long sur le passé pour y déceler des changements ou des constantes;

- Des personnes ayant une expérience professionnelle antérieure hors l'école primaire; et d'autres dont le métier d'enseignant est le premier et le seul exercé à ce jour; 
- Des personnes célibataires, d'autres vivant en couple; actuellement avec enfants ou sans enfants à charge.

Cinq professeurs des écoles ont été approchés de telle sorte que tous les critères de constitution de l'échantillon soient respectés au moins une fois.

\section{L'entretien semi-directif}

\section{La grille d'entretien}

La grille d'entretien sert la thématique suivante : De l'étudiant en Formation Initiale au professionnel d'aujourd'hui. Elle comporte quatre questions-repères, deux portant sur la formation initiale et deux sur la vie professionnelle. La première est censée provoquer un discours sur le vécu d'un contexte d'enseignement formel, et la seconde révéler quel statut le sujet accorde à la formation dans son parcours professionnel, ainsi que la manière dont il ressent l'exercice du métier.

\section{La formation initiale}

- Qu'est-ce qui vous a le plus marqué dans votre formation initiale au CFP?

- Qu'est-ce qui vous sert le plus de cette formation dans l'exercice de votre profession?

\section{Le milieu professionnel}

- Quelles sont pour vous les compétences majeures du professeur des écoles? 
- Qu'est-ce qui vous a aidé à acquérir de telles compétences? Phase conclusive

- Y a-t-il un sujet que nous n'aurions pas abordé, qui vous semble important ou qui vous tient particulièrement à cœur?

La dernière question - très ouverte - a souvent eu le même effet que les silences, soit la production d'un discours plus intimiste, la réponse s'exprimant d'ailleurs le plus souvent après un temps de réflexion significatif. Les entretiens ont tous évolué vers plus de détente. Ils ont été enregistrés et retranscrits intégralement.

\section{L’analyse de l'entretien : le symbolisme des termes}

\section{Les fondements de l'analyse}

G. Durand compte parmi les initiateurs des études de mythocritique, qui explorent les textes littéraires écrits (DURAND, G., 1961) ou oraux. S'agissant de l'analyse d'un entretien, l'étude du symbolisme des termes ne peut être assimilée à la mythocritique, dans la mesure où, d'une part l'oralité de ce texte relève du langage courant, et d'autre part, le but poursuivi est plus proche du dévoilement de tendances imaginaires que de celui de mythes individuels. La démarche s'en inspire toutefois, qui traque ce "'sens qui nous regarde' avec la vulgaire mimésis du fait divers (DURAND, G. 1996)». Avec la temporalité longue du discours sur la formation dans un parcours professionnel, se dessine une sorte de récit dont on peut dépasser l'aspect factuel avec les " essaims d'images » que les verbes et " gestes verbaux " révèlent. "La geste des héros immémoriaux et des dieux (DURAND, G. 1996)». " peut ainsi être approchée par la mise en valeur, notamment, des rapports du sujet au temps et à l'espace. Toutefois, la plurivalence du symbole s'oppose à un projet de vérité sur le texte - l'interprétation est 
inévitable et la toute-puissance du chercheur mise à mal. Un compromis « honnête " est cependant envisageable dans la recherche de redondances, qu'une « confrontation » avec les résultats de l'outil AT.9 pourra compléter ou relativiser.

\section{La mise en œuvre de l'analyse}

Le " matériel » de référence pour l'analyse du symbolisme des termes d'un entretien est, d'une manière générale, l'œuvre de G. Durand et, plus spécifiquement l'ouvrage intitulé « Les Structures Anthropologiques de l'Imaginaire (DURAND, G., 1969) ", dont le tableau isotopique des images s'est révélé particulièrement opératoire. L'exercice se déroule selon 3 phases, réalisées dans le même ordre pour chacun des sujets. Les deux premières étapes constituent des outils de travail que le dernier écrit synthétise et formalise. Dans un premier temps, déroulant la retranscription, nous avons observé, interprété et annoté chaque prise de parole du sujet au regard des divers " éléments imaginaires » significatifs de chacune des structures : les réflexes dominants (posturaux, copulatifs, digestifs), les schèmes verbaux (distinguer, relier, confondre), les archétypes épithètes et substantifs, les symboles. Nous avons également cherché à en repérer les "logiques » (exclusion - causalité - similitude). Il s'agissait d'objectiver les premières impressions, dans une première étape. Dans un second temps, pour limiter l'interprétation subjective du chercheur, nous avons « concrètement » classé toutes ces occurrences dans un texte, qui explique le tableau isotopique des images. On y trouve, entre autres, les rubriques «types de symboles » qui peuvent être "liés au temps : nyctomorphes ..» ou " relatifs à la maîtrise des peurs premières : ascensionnels, .. »-, ou encore, les axes selon lesquels les structures de l'imaginaire s'organisent - comme "la persévération et le redoublement ». Seul le premier entretien traité a fait l'objet d'un écrit spécifique pour cette 2ème étape. Par la suite, plus à l'aise avec les différents éléments de l'imaginaire, nous nous sommes d'emblée appuyée sur ce texte pour annoter les retranscriptions de manière plus précise et plus riche dans 
leurs marges. Le texte synthèse constitue la dernière étape de cette analyse. Il réorganise les données des deux premières phases du travail en rubriques thématiques sur la formation initiale et continue, le parcours professionnel et les conceptions de l'exercice du métier. Des éléments d'analyse sont repris plusieurs fois lorsqu'ils servent plusieurs thématiques. La conclusion esquisse le portrait " imaginaire » du sujet, qu'elle commence à envisager comme un personnage-héros.

Avec l'analyse du symbolisme des termes, il s'agit bien de traquer le sens caché du discours, par l'exploration de l'imaginaire que les supports du langage véhiculent. Dans cette démarche, impactée par le propre imaginaire du chercheur, la redondance fera office de garde-fou et de balise.

\section{AT.9 ou Archétype-Test à 9 éléments}

\section{Les principes de l'outil}

Dans le but d'obtenir des faits, qui puissent faire l'objet de comparaisons et de classifications, l'épreuve prend la forme d'un dessin à réaliser dans un cadre imparti, à partir de 9 archétypes - qui font fonction de stimuli symboliques. Cette production s'enrichit d'un récit écrit, qui précise notamment des données temporelles. L'ensemble est ensuite complété par un questionnaire.

\section{Choix des archétypes : fonctions et caractéristiques}

Dans le cartulaire de l'imaginaire élaboré par de G. Durand, Y. Durand a choisi les archétypes les plus aptes, d'une part à poser le problème du Temps et de la Mort et, d'autre part, à permettre la construction de solutions à ce problème. Selon G. Durand (DURAND, G., 1969), il existe trois catégories de symboles autour de l'angoisse : les symboles thériomorphes relatifs aux aspects anxiogènes de l'animalité -, les symboles nyctomorphes 
- se rapportant à l'eau, à la féminité et aux ténèbres inquiétantes -, et les symboles catamorphes - qui renvoient à la peur de la chute. Neuf éléments ont ainsi été sélectionnés, qui remplissent quatre fonctions complémentaires dans le modèle:

I. Un élément de dramatisation

Un élément de dramatisation est joué par l'un des trois êtres vivants proposés par le modèle : "le personnage ", sujet de l'action. Afin de ne pas induire la création d'un micro-univers de type héroïque, Y. Durand a volontairement employé un terme plus neutre que celui de «héros». Soumis à des données très personnelles du Sujet, « le personnage » permet à celui-ci la distance nécessaire à la création d'une histoire structurée.

II. Poser le problème de l'angoisse du temps

C'est avec les archétypes de « la chute » et du " monstre dévorant ", qu'Y. Durand pose le problème de l'angoisse du Temps Fatal. Le geste ou schème - de " la chute » s'actualise en archétype dans toutes les cultures - qu'il s'agisse de celle d'Icare dans la mer ou de Tantale dans les Enfers. D’une façon générale, elle traduit les échecs de l'homme, ses différentes pertes, à commencer par celle de l'équilibre qui constitue la première expérience de peur chez le nourrisson (MONTESSORI 1992, in DURAND, Y., 1988). L'expression "monstre dévorant » invite à une représentation très concrète des symboles thériomorphes. Quand l'animal domestique incarne des valeurs positives, d'autres animaux comme le reptile ou l'insecte véhiculent des valeurs négatives. De nouveau, le terme choisi pour susciter la représentation d'un être effrayant est relativement neutre. Le caractère monstrueux n'est pas réservé aux animaux réels, qui convient également aux animaux imaginaires comme le dragon et aux êtres humains. Quant au qualificatif " dévorant », il évoque le schème de l’avalage et la « gueule dentée » des animaux angoissants. 
III. Des « embrayeurs » de structuration

Trois éléments - "l'épée », "le refuge » et «quelque chose de cyclique (qui tourne, qui se reproduit ou qui progresse)»- sont utilisés comme des " embrayeurs " (DURAND, Y., 1988) de structuration. Ils montrent comment l'individu compose avec l'angoisse du temps. Dans la théorie de G. Durand, "l'épée » est un symbole diaïrétique référé aux structures schizomorphes du Régime Diurne de l'Imaginaire. Bien que n'ayant plus cours dans la vie quotidienne, l'arme continue de porter le sémantisme de la coupure, de la destruction, voire de la purification. L'archétype du "refuge » a été choisi pour appeler des réponses relevant du Régime Nocturne de l'Imaginaire et plus particulièrement de l'univers mystique, dans lequel le blottissement, l'intimité, et la recherche de protection dominent. Il se décline aisément au quotidien dans la prière, la fuite, la drogue ... Le troisième élément de cette catégorie vise à actualiser des images de l'univers synthétique du Régime Nocturne en ce qu'il doit conjointement satisfaire aux sémantismes cyclique et progressiste. Après avoir testé divers archétypes - la lune, la roue, l'arbre - qui ne se sont pas révélés de bons stimulus des structures synthétiques, Y. Durand a été « amené à retenir une expression ne préjugeant plus de l’image mais induisant le dynamisme cyclique, prolongé par un mouvement progressiste. D'où l'expression choisie : 'Quelque chose de cyclique (qui tourne, qui se reproduit et qui progresse)'»(DURAND, Y., 1988).

IV. Des éléments complémentaires

De par leur polyvalence symbolique, les trois archétypes " eau », « animal » et " feu » s'intègrent à n'importe quelle structure. Ils ont pour fonction d'en renforcer le sémantisme. L'eau est un archétype susceptible de présenter des aspects positifs comme des aspects négatifs. Elle est fatale lorsqu'elle coule indéfiniment, et lorsqu'elle se fait " 'image redoutable' du sang menstruel.» (Durand, Y., 1988). Symbole de limpidité et de pureté, elle s'intègre aux convergences d'images de type schizomorphe. Lorsqu'elle 
absorbe couleurs et éléments divers qui l'épaississent, elle devient « mystique ». Enfin, elle est «synthétique » par son cycle de transformation et dans ses fonctions fertilisantes.Chaque grande structure de l'Imaginaire s'actualise dans une ou plusieurs formes d'animaux. Les regroupements d'images de type schizomorphe sont renforcés par "l'oiseau ", que son aile apparente au mouvement ascensionnel, et qui réfère à des qualités morales plus qu'à son statut d'animal. Les compositions révélant une structure mystique utilisent le poisson, symbole d'avalage, qui permet de réduire l'angoisse dans un processus d'euphémisation de la dévoration par le monstre. Enfin, au service des structures synthétiques, le serpent « est le triple symbole de la transformation temporelle - [ dans la mue qui le change tout en lui permettant de rester lui-même] -, de la fécondité - [à la fois féminin car lunaire et symbole de virilité dans sa forme] et enfin de la pérennité ancestrale - [Ourobouros qui se mord la queue] ". Quand le feu - obtenu par percussion - sert les rites de purification, il se rattache aux constellations du Régime Diurne, aux structures schizomorphes. Il est isomorphe de la structure mystique du Régime Nocturne lorsqu'il participe de l'intimité dans la chaleur du foyer, et de la fusion des aliments par la cuisson. Obtenu par frottement, il s'apparente notamment aux symbolismes sexuels et aux symbolismes de renouvellement qui associent étroitement la vie et la mort. Il renforce alors les structures synthétiques.

\section{Elaboration de la liste des archétypes}

Ces 9 archétypes composent une liste organisée - « une chute, une épée, un refuge, un monstre dévorant, quelque chose de cyclique (qui tourne, qui se reproduit ou qui progresse), un personnage, de l'eau, un animal (oiseau, poisson, reptile ou mammifère), du feu. » - dans laquelle l'ordre des termes ne relève pas du hasard. C'est pour répondre à la tradition judéo-chrétienne - «Au début était la chute » - que la liste s'ouvre par l'archétype du même nom. On peut lire sans virgule de séparation : «Le monstre dévorant quelque chose de cyclique ». A la fois « promesse de renouveau » et « médiation entre les dieux et les Hommes 
»(DURAND, Y., 1988), à la fois ouverture et lien, « le feu » clôture cette liste. Certaines juxtapositions de mots ont été évitées, qui pouvaient induire des groupements graphiques. Par exemple : «personnage - épée - monstre dévorant » ou «personnage - refuge - feu ». La structuration s'organise autour des 6 premiers archétypes, les trois derniers ne jouant qu'un rôle secondaire.

\section{Protocole de passage du test}

Le recueil des messages symboliques s'effectue selon un protocole réglementé, dont les différentes phases sont indiquées et réparties sur les pages d'une feuille à dessin double, de format $21 \times 27 \mathrm{~cm}$, présentée horizontalement. L'épreuve s'effectue sans gomme ni règle, avec « un crayon de dureté $n^{\circ} 2$ bien taillé » (DURAND, Y., 1988).

Etape 1. Après s'être identifié sur la première page du document, le sujet prend connaissance du texte de présentation de l'épreuve qui y figure. Une minute suffit à cela.

Etape 2. Dans l'épreuve qui suit, vous aurez à composer un dessin à partir de neuf éléments qui vous seront préalablement donnés. Ensuite il vous sera demandé un récit qui raconte ce qui se passe dans votre dessin. Enfin vous serez invité à répondre à un questionnaire qui précisera encore dessin et récit.

Dans un second temps, le sujet prend connaissance des neuf éléments archétypaux supports de sa composition graphique et qui figurent au verso de la première page. Selon Y. Durand, "l'essentiel de l'élaboration créatrice structurante " est assurée par cette représentation relativement spontanée. L'expérimentateur ne donne jamais d'exemple de représentation. Son but est de faire réaliser un dessin organisé et non une juxtaposition d'éléments isolés. Si besoin, il réaffirme au sujet la nécessité de représenter chaque archétype à sa convenance - aucune conformation à un modèle n'est attendue - dans la mesure où le dessin s'intègre à la scène qu'il veut figurer. Il propose en revanche, systématiquement dans 
le troisième temps du protocole, une reformulation de la consigne : « Il s'agit d'une épreuve d'imagination; même si vous ne savez pas dessiner, cela ne fait rien. Il n'y a pas de mauvaises réponses dans ce test. Pour l'animal, choisissez celui que vous voulez, celui qui s'adapte le mieux à votre composition. N'oubliez pas que vous devez utiliser tous les éléments qui vous sont proposés. Cependant vous pouvez en ajouter d'autres si vous voulez. Lorsque vous aurez terminé votre dessin, vous ferez un récit pour expliquer, pour raconter ce qui se passe dans votre composition. Vous pouvez revenir à la première afin de relire, si vous le désirez, les consignes du travail qui vous est demandé. (DURAND, Y., 1988, p.71)».

Composez un dessin avec: une chute, une épée, un refuge, un monstre dévorant, quelque chose de cyclique (qui tourne, qui se reproduit ou qui progresse), un personnage, de l'eau, un animal (oiseau, poisson, reptile ou mammifère), du feu. Les $30 \mathrm{mn}$ allouées à l'épreuve complète - dessin et récit d'explicitation - peuvent être prolongées si le sujet en fait la demande.

Etape 3. Sur la troisième page du document, le Sujet est invité à raconter - à la manière d'un conte ou d'un mythe - l'histoire dont son dessin est le support. L'exercice sert un "processus_rationalisant de mise en ordre temporel et parfois même de justification de la représentation figurée " (DURAND, Y., 1988).

Etape 4. Suite à la réalisation des deux premiers exercices, un questionnaire est proposé qui permettra d'en compléter les informations. Répondez avec précision aux questions suivantes :

a) Autour de quelle idée centrale avez-vous construit votre composition ? Avez-vous hésité entre deux ou plusieurs solutions ? Si oui, lesquelles?

b) Par quoi (lecture, film, etc.) avez-vous été éventuellement inspiré ?

c) Indiquez parmi les 9 éléments de votre composition :

d) C1) Les éléments essentiels autour desquels vous avez bâti votre construction ; 
e) C2 Les éléments que vous auriez éliminés. Pourquoi ?

f) Comment se termine la scène que vous avez imaginée?

g) Si vous deviez, vous participer à la scène que vous avez composée, où seriez-vous? Que feriez-vous?

Etape 5. Un tableau de classement de chaque archétype est proposé, où il s'agit d'expliquer quelle représentation le sujet lui a donnée, quel rôle il lui a conféré dans son récit, et ce qu'il symbolise. En classant images, rôles et symbolismes des 9 éléments, le Sujet révèle la manière dont il a transcrit les symboles sur le plan linguistique.

Ce tableau est proposé vierge, mais le chercheur peut apporter son aide en donnant un exemple de réponse aux questions suivantes :

- pour la colonne A (représentation) : "Par quoi avez-vous représenté l'élément $X$ ? ",

- pour la colonne B (rôle) : "Quel est le rôle, la raison d'être de l'élément X?",

- pour la colonne C : "Que symbolise, que représente, que signifie pour vous l'élément?».

Il est attendu de l'AT.9 un dessin unifié, à partir d'archétypes qui ne possèdent aucun lien entre eux dans leur présentation. L'analyse vise à faire apparaître des groupements isomorphes de symboles, des micro-univers mythiques représentatifs du fonctionnement de l'imaginaire selon la théorie de G. Durand.

\section{La mise en œuvre de l'analyse}

Les 5 AT.9 sont soumis à un même protocole d'analyse, dont la première étape consiste à décrire la production graphique aussi fidèlement et objectivement que possible, tout en accueillant 
pourtant les quelques éléments d'interprétation qui s'imposeraient. Cette description renseigne sur la forme générale du tableau, celles du personnage principal et des éventuels acteurs secondaires, sur la nature des différents éléments, leur organisation spatiale, ceux qui sont mis en valeur par leurs proportions ou parce que plus marqués par le crayon. Une thématique imaginaire s'en dégage, que la suite du travail, par un va-et-vient continu entre production graphique, récit - forme et fond - et questionnaire, cherche à préciser et à réajuster. Chacun des 9 archétypes est ainsi passé au crible de ses composantes morphologiques, fonctionnelles et symboliques mises au regard de leur portée imaginaire. Un document écrit sous forme de tableau rend compte de ces données directement déduites de l'expérimentation de Durand, Y. (1988). L'analyse, qui prend en compte les redondances imaginaires, les regroupements d'images, est finalement formalisée dans un texte synthèse de 3 à 4 pages. Elle n'est pas retouchée par les résultats de la restitution-réception, lesquels seront intégrés aux étapes ultérieures qui mettent en lien les informations produites par les différents outils méthodologiques.

\section{Le retour aux intéressés : la restitution-réception de I'AT.9}

La restitution, aux intéressés, des propositions d'analyse de leur AT.9 s'appuie sur les travaux de B. Bergier (BERGIER, B., 2000), lesquels considèrent le sujet "comme étant à la fois objet à connaître et objet connaissant ». Avec elle, l'exploration " clinique » de l'imaginaire des professeurs des écoles enjoints à l'apprenance se poursuit. Chaque enseignant ayant sollicité un avis sur sa production sitôt celle-ci achevée, des impressions ont été livrées, avec la promesse du retour écrit d'une analyse plus formelle. D'autre part, Marielle allant jusqu'à dire "C'est parce que c'est toi, sinon je ne le ferais pas, c'est très engageant car très intime.". 


\section{Les conditions de la restitution-réception}

Le respect de la clause de confidentialité a induit des restitutions individuelles. Selon les sujets, l'exercice a fait l'objet d'une seconde ou une troisième rencontre, la méthodologie déroulant trois temps distincts entretien, AT.9, restitution - dont les deux premiers sont parfois réalisés à la suite. Pour chaque professeur des écoles, les diverses rencontres se sont déroulées dans le lieu qu'elles avaient choisi la première fois : l'école de Claire et de Marielle, le domicile de Marie, Maurice et Robert. Les précautions oratoires et l'objectif de l'exercice posés, le sujet est invité à retrouver son AT.9 - dessin, récit et réponses au questionnaire - puis à prendre connaissance de nos propositions d'analyse. La dernière phase de la rencontre est enregistrée avec son accord. Il y est invité à s'exprimer librement à partir de ces deux lectures. Certaines relances sont parfois nécessaires : "Est-ce que quelque chose t'a choquée? ", "Veux-tu me dire ce qui t'a fait rire? ", "Et si tu devais changer quelque chose dans ton dessin ou ton récit, qu'est-ce que tu modifierais? ", "Que proposes-tu pour l'interprétation en termes de modifications ou de compléments? ». De longs silences s'installent parfois, tandis que les yeux du sujet vont et viennent, de sa production à la nôtre. Pour le chercheur, "Se taire, c'est accepter d'entendre l'interlocuteur de terrain statuer sur la plausibilité des données factuelles rapportées, sur la vraisemblance de l'interprétation sociologique [...]. (BERGIER, B., 2000)»

\section{Précautions oratoires}

Après avoir présenté la démarche de restitution-réception, nous avons redonné à certains termes du langage courant leur signification particulière dans le test de Y. Durand.

Une question d'éthique. Envisager ce retour vers le sensible, vise, dans un premier temps, à désacraliser la parole du chercheur, "c'est refuser que [l'interlocuteur de terrain] fasse le mort, que sa voix s'éteigne ou soit couverte par la toute-puissance du modélisateur (BERGIER, B., 2000)». 
Nos premières conclusions sur la définition des micro-univers individuels sont très prudentes, d'une part parce que le doute est un compagnon du chercheur, d'autre part parce que nous sommes inexpérimentée, et enfin, parce que l'approche théorique retenue nous y incite. La richesse des travaux de G. Durand et de Y. Durand nécessite une maturation pour appropriation, qui nous interdit de prétendre en maîtriser tous les aspects. Le regard critique des intéressés est susceptible d'enrichir et nuancer nos propositions d'interprétation et d'en lever les éventuelles ambiguïtés.

Clarification sémantique. "La matière langagière qui n'est pas directement accessible aux interlocuteurs de terrain est l'objet d'un travail de traduction (BERGIER, B., 2000)». Le champ de l'imaginaire emprunte au langage courant des termes qu'il dote d'une signification particulière. Il en va ainsi des qualificatifs des micro-univers - tout à tour " héroïques ", " mystiques ", " purs ", " impurs ", au symbolisme " positif » ou " négatif » -, qui, d'ordinaire sont affectivement connotés et qui, dans ce contexte, renseignent sur la convergence des symboles, ainsi que sur la performance et le sentiment de sécurité du personnage de l'AT.9.

A l'exigence de clarification sémantique, s'ajoute la nécessité d'identifier « l'objet » qualifié, qui n'est pas le sujet lui-même, mais l'univers mythique que sa production révèle. L'analyse de l'AT.9 produit par un sujet n'est pas un exercice de jugement de sa personne.

Le caractère "non pérenne » des résultats du test. Nous avons de nouveau positionné l'AT.9 comme un test valable à un moment donné, susceptible d'être différent s'il était réalisé à un autre moment, dans un autre contexte.

C'est seulement à la condition d'un « bien-entendu " consécutif à l'ensemble de ces clarifications, que nous pourrons nous engager, sujet et chercheur, dans un débat cognitif mais pas affectif - bien que non dénué d'affectivité -, ni thérapeutique - bien que de type clinique. En effet, écrit Bertrand Bergier (2000, p.130), "Si la démarche se donne des fins de connaissance, le dialogue n'est jamais purement cognitif. L'altérité d'Artémis a pour compagnon de route Dyonisos ». 


\section{Exploration de l'imaginaire des professeurs des écoles.}

\section{Repères méthodologiques}

\begin{tabular}{|c|c|c|c|c|c|c|}
\hline $\begin{array}{l}\text { REGIMES OU } \\
\text { POLARITÉS }\end{array}$ & \multicolumn{2}{|l|}{ DIURNE } & \multicolumn{4}{|l|}{ NOCTURNE } \\
\hline STRUCTURES & \multicolumn{2}{|c|}{$\begin{array}{l}\text { SCHIZOMORPHES } \\
\text { (ou Héroïques) } \\
\text { 1.idéalisation et recul « autistique ». } \\
\text { 2.diaïrétisme (spaltung). } \\
\text { 3.géométrisme, symétrie, gigantisme. } \\
\text { 4.antithèse polémique. }\end{array}$} & \multicolumn{2}{|c|}{$\begin{array}{l}\text { SYNTHETIQUES } \\
\text { (ou Dramatiques) } \\
\text { 1.coincidentis oppositorum et } \\
\text { systématisation. } \\
\text { 2.dialectique des } \\
\text { antagonistes, dramatisation. } \\
\text { 3.historisation. } \\
\text { 4.progressisme partiel (cycle) } \\
\text { ou total }\end{array}$} & \multicolumn{2}{|c|}{$\begin{array}{l}\text { MYSTIQUES } \\
\text { (ou Antiphrasiques) } \\
\text { 1.redoublement et } \\
\text { persévération } \\
\text { 2.viscosité, adhésivité } \\
\text { antiphrasique } \\
\text { 3.réalisme sensoriel } \\
\text { 4.mise en miniature (Gulliver) }\end{array}$} \\
\hline $\begin{array}{l}\text { PRINCIPES } \\
\text { d'explication et } \\
\text { de justification } \\
\text { ou LOGIQUES }\end{array}$ & \multicolumn{2}{|c|}{$\begin{array}{l}\text { Représentation objectivement } \\
\text { hétérogénéisante (antithèse) et } \\
\text { subjectivement homogénéisante } \\
\text { (autisme). Les Principes d'EXCLUSION, } \\
\text { de CONTRADICTION, d'IDENTITE, } \\
\text { jouent à plein }\end{array}$} & \multicolumn{2}{|c|}{$\begin{array}{l}\text { Représentation diachronique } \\
\text { qui relie les contradictions par } \\
\text { le facteur temps. Le Principe } \\
\text { de CAUSALITÉ, sous toutes } \\
\text { ses formes (spéc. FINALE et } \\
\text { EFFICIENTE) joue à plein. }\end{array}$} & \multicolumn{2}{|c|}{$\begin{array}{l}\text { Représentation objectivement } \\
\text { homogénéisante } \\
\text { (persévération) et } \\
\text { subjectivement } \\
\text { hétérogénéisante (effort } \\
\text { anti-phrasique). Les Principes } \\
\text { d'ANALOGIE, de SIMILITUDE } \\
\text { jouent à plein }\end{array}$} \\
\hline $\begin{array}{l}\text { REFLEXES } \\
\text { DOMINANTS }\end{array}$ & \multicolumn{2}{|c|}{$\begin{array}{l}\text { Dominante POSTURALE avec ses } \\
\text { dérivés manuels et l'adjuvant } \\
\text { des sensations à distance (vue, } \\
\text { audiophonation) }\end{array}$} & \multicolumn{2}{|c|}{$\begin{array}{l}\text { Dominante COPULATIVE } \\
\text { avec ses dérives moteurs } \\
\text { rythmiques et ses adjuvants } \\
\text { sensoriels (Kinésiques, } \\
\text { musicaux-rythmiques, etc.) }\end{array}$} & \multicolumn{2}{|c|}{$\begin{array}{l}\text { Dominante DIGESTIVE } \\
\text { avec ses adjuvants } \\
\text { caenesthésiques, thermiques } \\
\text { et ses dérivés tactiles, } \\
\text { olfactifs, gustatifs. }\end{array}$} \\
\hline \multirow{2}{*}{$\begin{array}{l}\text { SCHEMES } \\
\text { "VERBAUX " }\end{array}$} & \multicolumn{2}{|l|}{ DISTINGUER } & \multicolumn{2}{|l|}{ RELIER } & \multirow{2}{*}{\multicolumn{2}{|c|}{$\begin{array}{l}\text { CONFONFRE } \\
\rightarrow \text { Descendre, posséder, } \\
\text { pénétrer }\end{array}$}} \\
\hline & $\begin{array}{l}\text { Séparer } \neq \\
\text { Mêler; }\end{array}$ & Monter $\neq$ Chuter $\leftarrow$ & $\begin{array}{l}\text { Mûrir } \\
\rightarrow \text { Progresser }\end{array}$ & $\begin{array}{l}\text { Revenir } \\
\text { Recenser } \leftarrow\end{array}$ & & \\
\hline $\begin{array}{l}\text { ARCHETYPES } \\
\text { "EPITHETES » }\end{array}$ & $\begin{array}{l}\text { Pur } \neq \text { Souillé } \\
\text { Clair } \neq \\
\text { Sombre }\end{array}$ & Haut $\neq$ Bas & $\begin{array}{l}\text { En avant } \\
\text { Avenir }\end{array}$ & $\begin{array}{l}\text { Arrière } \\
\text { Passé }\end{array}$ & \multicolumn{2}{|c|}{$\begin{array}{l}\text { Profond, Calme, Chaud, } \\
\text { intime, Caché }\end{array}$} \\
\hline $\begin{array}{l}\text { Situation des } \\
\text { "catégories » du } \\
\text { jeu de TAROTS }\end{array}$ & $\begin{array}{l}\text { LE } \\
\text { GLAIVE } \leftarrow\end{array}$ & $\rightarrow$ LE SCEPTRE $\leftarrow$ & $\begin{array}{l}\rightarrow \text { LEE } \\
\text { BÂTON }\end{array}$ & $\begin{array}{l}\rightarrow \text { LEE } \\
\text { DENIER } \leftarrow\end{array}$ & \multicolumn{2}{|l|}{$\begin{array}{l}\rightarrow \mathrm{LA} \\
\text { COUPE }\end{array}$} \\
\hline $\begin{array}{l}\text { ARCHETYPES } \\
\text { "SUBSTANTIFS » }\end{array}$ & $\begin{array}{l}\text { La Lumière } \neq \\
\text { Les Ténèbres } \\
\text { L’Aire } \neq \text { Le } \\
\text { Miasme } \\
\text { L’Arme } \\
\text { Héroïque } \neq \\
\text { Le Lien } \\
\text { Le Baptême } \\
\text { ₹ La } \\
\text { Souillure }\end{array}$ & $\begin{array}{l}\text { Le Sommet } \neq \text { Le } \\
\text { Gouffre. } \\
\text { Le Ciel } \neq \text { L'Enfer. } \\
\text { Le Chef } \neq \text { L'Inférieur. } \\
\text { Le Héros } \neq \text { Le } \\
\text { Monstre. } \\
\text { L'Ange } \neq \text { L'Animal. } \\
\text { L'Aile } \neq \text { Le Reptile }\end{array}$ & $\begin{array}{l}\text { Le } \\
\text { Feu-flamme } \\
\text { Le Fils } \\
\text { L'Arbre } \\
\text { Le Germe }\end{array}$ & $\begin{array}{l}\text { La Roux } \\
\text { La Croix } \\
\text { La Lune } \\
\text { L'Androgyne } \\
\text { Le Dieu Pluriel }\end{array}$ & $\begin{array}{l}\text { Le } \\
\text { Microcosme } \\
\text { L'Enfant } \\
\text { Le Poucet } \\
\text { L'Animal } \\
\text { Gigogne } \\
\text { La Couleur } \\
\text { La Nuit } \\
\text { La mère } \\
\text { Le Récipient }\end{array}$ & $\begin{array}{l}\text { La Demeure } \\
\text { Le Centre } \\
\text { La Fleur } \\
\text { La Femme } \\
\text { La } \\
\text { Nourriture } \\
\text { La Substance }\end{array}$ \\
\hline $\begin{array}{l}\text { Des Symboles au } \\
\text { Synthèmes }\end{array}$ & $\begin{array}{l}\text { Le Soleil, } \\
\text { l'Azur L'œil } \\
\text { du Père Les } \\
\text { Runes Le } \\
\text { Mantra Les } \\
\text { Armes La } \\
\text { Clôture La } \\
\text { Circoncision } \\
\text { La Tonsure } \\
\text { etc. }\end{array}$ & $\begin{array}{l}\text { L'Echelle, L'Escalier, } \\
\text { Le Bétyle, Le Clocher, } \\
\text { La Ziqqurat, L'Aigle, } \\
\text { L'Alouette, La } \\
\text { Colombe, Jupiter, etc. }\end{array}$ & \multicolumn{2}{|c|}{$\begin{array}{l}\text { Le Calendrier, L'Arithmologie, } \\
\text { La Triade, La Tétrade, } \\
\text { L'Astrobiologie L'initiation Le } \\
\text { Sacrifice L'Orgie Le Dragon Le } \\
\text { Messie La spirale La Pierre } \\
\text { philosophale L'Escargot La } \\
\text { Musique etc. }\end{array}$} & $\begin{array}{l}\text { Le Ventre } \\
\text { Avaleurs et } \\
\text { Avalés Osiris } \\
\text { Les Teintures } \\
\text { Les Gemmes } \\
\text { Mélusine } \\
\text { Le Voile Le } \\
\text { Manteau La } \\
\text { Coupe etc. }\end{array}$ & $\begin{array}{l}\text { La Tombe } \\
\text { Le Berceau } \\
\text { L'Ile La } \\
\text { Caverne Le } \\
\text { Lait Le Vin } \\
\text { l'Or Le Miel } \\
\text { etc. }\end{array}$ \\
\hline
\end{tabular}




\section{Schéma de la méthodologie}

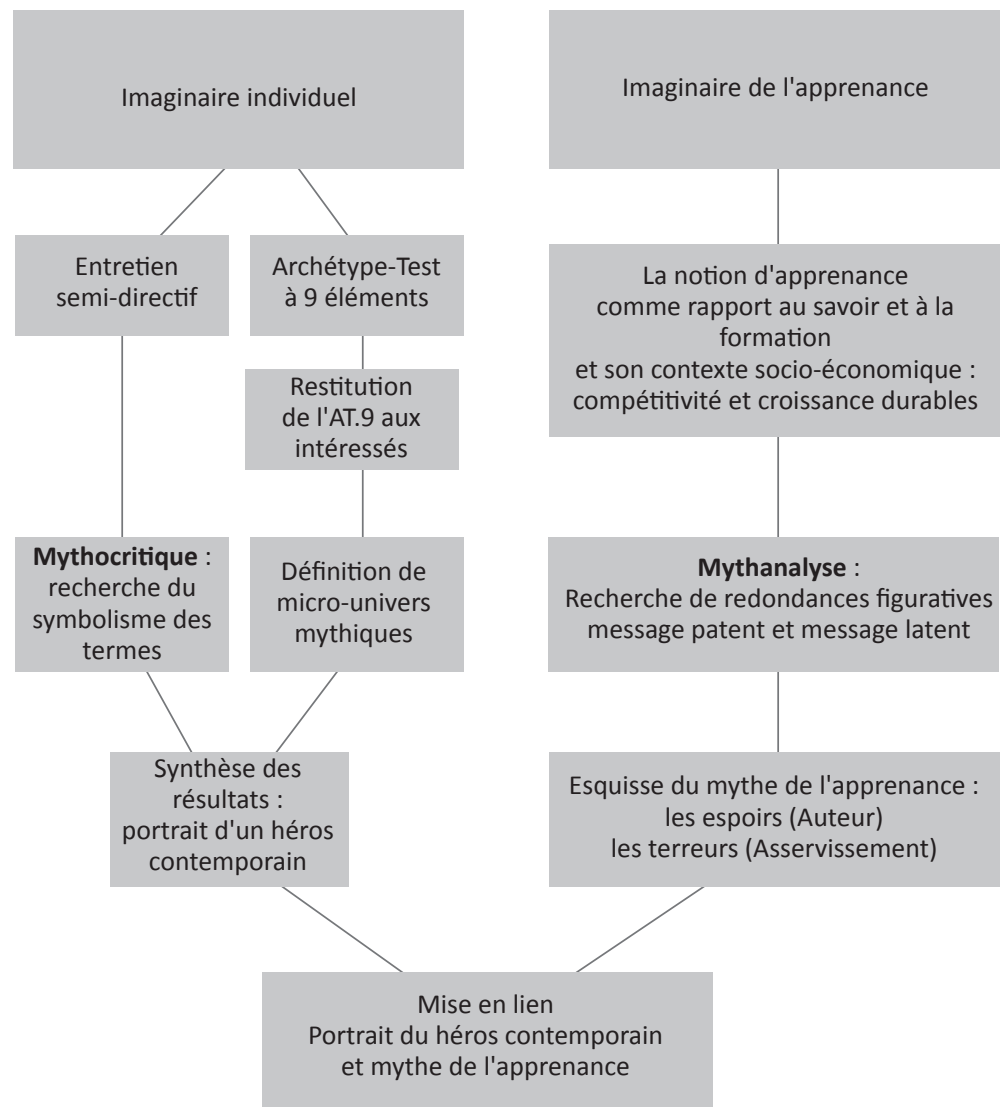




\section{Références :}

BACHELARD, G. L'eau et les rêves. Corti: Paris, 1942.

BACHELARD, G. L'air et les songes. Corti: Paris, 1943.

BEILLEROT, J. La société pédagogique. Paris: PUF, 1982, 223 p.

BERGIER, B. Repères pour une restitution de la recherche en Sciences Sociales. Paris: L'Harmattan, 2000, 302 p.

CARRE, P. L'apprenance: Vers un nouveau rapport au savoir. Paris: Dunod, 2005, $212 \mathrm{p}$.

CEE. Enseigner et apprendre: Vers la société cognitive. Luxembourg: Office des publications officielles des Communautés Européennes, 1995, 142 p.

CHARRIER, M-C. Imaginaire des professeurs des écoles et apprenance. Thèse de Doctorat. Université de Nantes, 2012. Inédite.

COHEN, D. cité par P. Carre. In: CARRE, P. L'apprenance: Vers un nouveau rapport au savoir. Paris: Dunod, 2005. 212 p.

DERRIDA, J. De la grammatologie. Paris: Les Editions de Minuit, 1967.

DUMAZEDIER, J. Penser l'autoformation: société d'aujourd'hui et pratiques d'autoformation. Lyon: Chronique sociale, 2002, 172 p.

DUMAZEDIER, J. "La société éducative et ses incertitudes". In: Éducation permanente. n. 44, Oct 1978.

DURAND, G. "Une cartographie de l'imaginaire"(interview). In: Sciences Humaines. n. 90, Janvier, 1999.

DURAND, G. Champs de l'imaginaire. (org. CHAUVIN, D.). Grenoble: ELLUG, 1996, $262 \mathrm{p}$.

DURAND, G. Introduction à la mythodologie: Mythes et sociétés. Paris: Albin Michel, 1996, $243 \mathrm{p}$.

DURAND, G. Les structures anthropologiques de l'imaginaire. Paris: Dunod, 1992, $536 \mathrm{p}$. 
DURAND, G. L'imagination symbolique. Paris: Puf, 1964, 132 p.

DURAND, G. Le Décor mythique de la Chartreuse de Parme. Paris: Jose Corti, 1961, $256 \mathrm{p}$.

DURAND, Y. Une technique d'étude de l'imaginaire: l'AT.9. Paris: l'Harmattan, 2005, $280 \mathrm{p}$.

DURAND, Y. "L'apport de la perspective systémique de Stéphane Lupasco à la théorie des structures de l'imaginaire et à son expérimentation". In: Bulletin Interactif du Centre International de Recherches et Études transdisciplinaires. n. 13, mai 1998. Disponível em: 〈http://nicol.club.fr/ciret/bulletin/b13/b13c3.htm〉.

DURAND, Y. L'exploration de l'Imaginaire, introduction à la modélisation des Univers Mythiques. Paris: L’espace bleu. 1988, 354 p.

FABRE, M. Eduquer pour un monde problématique: La carte et la boussole. Paris: Puf, 2011. 220p.

FABRE, M. "Formation et modernité chez Bachelard: entre la joie d'apprendre et le bonheur d'habiter”. In: Le Télémaque. n. 15. maio 1999.

FOUCAULT, M. Les mots et les choses: une archéologie des sciences humaines. Paris: Editions Gallimard, 1966.

FREUD, S. Essais de psychanalyse. Paris: Payot, (1968). 280 p.

FREUD, S. CEuvres complètes, psychanalyse, volume 4: L'interprétation des rêves. Paris: Puf, 1900.

GIORDAN, A. Apprendre!. Paris: Belin, 1998, 254 p.

JUNG, C. G. Psychologie de l'inconscient. Paris: Le Livre de poche, 1995.

JUNG, C. G. L’homme et ses symboles. Paris: Robert Laffont, 1964.

KAËS, R. Crise, rupture et dépassement. Paris: Dunod, 1997, 291 p.

LACAN, J. "Fonction et champ de la parole et du langage en psychanalyse". In: $L a$ psychanalyse. n. 1, Sur la parole et le langage, 1956, p. 81-166.

LEVI-STRAUSS, C. Les structures élémentaires de la parenté. Paris: Puf, 1949.

LUPASCO, S. Qu'est-ce qu'une structure?. Paris: Christian Bourgois, 1967. 
MAFFESOLI, M. Iconologies. Paris: Editions Albin Michel, 2008, 246 p.

MONTESSORI, M. l'Enfant. Paris: Desclée De Brouwer, 1992, 202 p. In: DURAND, Y. L'exploration de l'Imaginaire, introduction à la modélisation des Univers Mythiques. Paris: L'espace bleu, 1988, 354 p.

PASCAL, B. Pensées, Les dangers de l'imagination: fragment 78 (1669). Paris: Édition Lafuma 44, 1993.

PINEAU, G. Produire sa vie, autoformation et autobiographie. Montréal: Editions Saint Martin, Paris: Edilig, 1983, 419 p.

QUIVY, R.; van CAMPENHOUDT, L. Manuel de recherche en sciences sociales. Paris: Dunod, 1988, 1995, 2006.

RICOEUR, P. Le conflit des interprétations: Essais d'herméneutique. Paris: Editions du Seuil, 1969, 662 p.

RINAUDO, J.L. "Construction identitaire des néo enseignants". In: Recherche et formation. n. 47, 2004, p. 141-153.

ROCHA-PITTA, D. "Fractalité et nouvelles constellations mythiques au Brésil". In: Loxias. v. 2. 15 jan 2004. Disponível em: <http://revel.unice.fr/loxias/document. html?id=872>.

SAUSSURE, F. Cours Linguistique Générale. Paris: Payot, 1916. (1916 póstuma).

SIBONY, D. Entre-Deux: l'origine en partage. Paris: Ed. du Seuil, 1991, 398 p.

TROCME-FABRE, H. Réinventer le métier d'apprendre. Paris: Edition d'Organisation, 1999, $269 \mathrm{p}$.

Recebido: 20/07/2015

Received: 07/20/2015

Aprovado: 11/10/2015

Approved : 10/11/2015 